\title{
Catastrophic Antiphospholipid Syndrome
} in a Patient with Immune Thrombocytopenic Purpura

\author{
Seby JOHN ${ }^{1}$, Keith McCRAE ${ }^{2}$ \\ ${ }^{1}$ Cleveland Clinic, Department of Internal Medicine \\ ${ }^{2}$ Cleveland Clinic, Taussig Cancer Institute and Department of Cell Biology, OHIO, USA
}

\section{CASE PRESENTATION}

A 47-year old Caucasian male with 7-year history of Immune Thrombocytopenic Purpura (ITP) presented with acute onset pain and purplish-blue discoloration of the left fifth toe and pleuritic chest pain. At the time of ITP diagnosis, he was positive for lupus anticoagulant (LA) and high titer of IgG anticardiolipin antibodies (aCL). However, his platelet count had previously remained stable at approximately $40,000 / \mu \mathrm{L}$ and he never required treatment. His examination was normal except for dusky cyanosis and coolness of the left fifth toe associated with extreme tenderness. Abnormal laboratory studies on admission included leukocytosis $(11.610 / \mu \mathrm{L})$, thrombocytopenia $(76,000 / \mu \mathrm{L})$ and elevated D-dimer (770 ng/mL FEU). Cardiac enzymes, EKG and Chest X-ray were normal. A lower extremity arterial study done prior to admission was normal.

Given his prior antiphospholipid antibody (APLA) positivity, thromboemboli to the toe and possible pulmonary embolus (PE) was suspected, for which unfractionated heparin was started. However, spiral chest CT was negative for PE. Other studies including a transthoracic and transesophageal echocardiogram, Holter monitoring, CT aorta and venous duplex studies were all normal. Laboratory studies confirmed persistent positivity for APLA, with an aPTT of $55.7 \mathrm{sec}$ (normal PT/INR), positive LA, elevated aCL, (IgG>150 GPL; IgM 36 MPL; IgA 27 APL) and elevated $\beta_{2}$-glycoprotein 1 antibodies (IgG 76 SGU; IgM 25 SMU).

One week after admission, the patient became hypotensive, and was diagnosed with a non-ST segment myocardial infarction (NSTEMI). He underwent emergent cardiac catheterization which did not show angiographically significant disease, except for minimal myocardial blush and sluggish flow in the left circumflex distribution. Concomitantly, he developed acute elevation of his liver enzymes and worsening renal function. A renal vascular duplex scan did not show renal artery or venous occlusion. Complaints of persistent pleuritic chest pain led to a lung ventilation-perfusion scintigraphy scan that revealed an intermediate probability of PE. With this constellation of symptoms with no apparent unifying cause, the patient was diagnosed with Catastrophic Antiphospholipid Syndrome (CAPS) and plasma exchange was initiated. He received three exchanges which led to normalization of the cardiac enzymes, liver function tests and decreased creatinine levels. Indefinite anticoagulation was recommended. 


\section{DISCUSSION}

CAPS is a life-threatening complication which occurs in less than $1 \%$ of patients with Antiphospholipid Syndrome (APS). Patients with CAPS present with 1) evidence of multi-organ involvement developed over a very short interval; 2) histopathologic evidence of multiple small-vessel occlusions; and 3) laboratory confirmation of APLA usually in high titer. ${ }^{1,2}$ Our patient satisfied the criteria for diagnosis of probable CAPS. ${ }^{3} \mathrm{He}$ had involvement of the heart (NSTEMI), lung (PE), kidney (AKI), liver, and toe all within one week, with presence of APLA in high titers. It is likely that this patient had microangiopathic occlusive small vessel disease of the above organs unlike the typical large vessel-type occlusions seen in the simple APS.

Thrombocytopenia is frequently found in patients with the APS, with a reported incidence of $22-42 \%$ in different series. Indeed, it has recently been suggested that thrombocytopenia might be considered as one of the clinical manifestations of the antiphospholipid syndrome. ${ }^{4}$ Putative mechanisms include direct binding of APLA to platelet phospholipids, concomitant anti-platelet glycoprotein antibodies, and platelet activation and/or damage leading to accelerated clearance. Conversely, a high percentage of patients with ITP also have APLA, with a reported incidence of $25-75 \%$. However, the clinical significance of this observation remains uncertain. Stasi et al..$^{5,6}$ found aCL and/or LA in $46 \%$ of 149 patients with newly diagnosed ITP, though the rate of thrombotic events was not higher in APLApositive patients. Because of this observed lack of clinical association between APLA and ITP, the American Society of Hematology ITP Practice and Guideline Panel did not recommend routine measurement of APLA in patients with ITP.?

However, two subsequent studies have reported an increased risk of thrombosis in ITP patients with APLA at diagnosis. Diz-Kucukkaya et al ${ }^{8}$ followed 82 patients with ITP and observed a much higher thrombosis rate on 5 year follow-up (60\% vs. $2 \%$ ). The risk of thrombosis was strongly associated with the presence of a lupus anticoagulant. No differences were found between APLA positive and negative patients in terms of ITP characteristics and/or response to treatment. Similarly, Pierrot-Deseilligny et al. ${ }^{9}$ followed 215 ITP adults for a median of
31 months, noting that $7 \%$ of patients with APLA developed thrombosis. Similar to the previous study, thrombosis was associated with lupus anticoagulant, as well as a high IgG-aCL level.

Much controversy has existed over the clinical significance of APLA in ITP patients, but the above studies strongly suggest that an increased risk of thrombosis may exist. Unlike systemic lupus erythematosus, which can be followed clinically given its slow progression, APS complications are unpredictable. Therefore, this report suggests that serologic testing for APLA should be performed in patients with ITP, and if positive (especially LA), such individuals should be followed with heightened awareness of the potential for thrombotic events, including CAPS. Indeed, such follow up may be warranted given the increased risk of thrombosis suggested in population-based studies of ITP. ${ }^{10}$ While previous guidelines recommended that evaluating ITP patients for APLA was unnecessary, the evidence presented above suggest that this recommendation be reconsidered.

\section{REFERENCES}

1. Asherson RA, Cervera R, Piette JC, et al. Catastrophic antibody syndrome. Clinical and laboratory features of 50 patients. Medicine (Baltimore) 77: 195-207, 1998..

2. Asherson RA, Cervera R, Piette JC, et al. Catastrophic antiphospholipid syndrome: clues to the pathogenesis from a series of 80 patients. Medicine (Baltimore) 80: 355-376, 2001.

3. Asherson RA, Cervera R, de Groot P, et al. Catastrophic antiphospholipid syndrome: International consensus statement on classification criteria and treatment guidelines. Lupus 12: 530-534, 2003.

4. McCrae KR. Blind men and the APS. Blood 116: 2871-2873, 2010.

5. Stasi R, Stipa E, Masi M, et al.: Prevalence and clinical significance of elevated antiphospholipid antibodies in patients with idiopathic thrombocytopenic purpura. Blood 84: 4203-4208, 1994.

6. Stasi R, Stipa E, Masi M, et al. Long-term observation of 208 adults with chronic thrombocytopenic purpura. Am J Med 98: 436-442, 1995.

7. George JN, Woolf SH, Raskob GE, et al. Idiopathic thrombocytopenic purpura: a practice guideline developed by explicit methods for the American Society of Hematology. Blood 88: 3-40, 1996. 
8. Diz-Kucukkaya R, Hacihanefioglu A, Yenerel M, et al. Antiphospholipid antibodies and antiphospholipid syndrome in patients presenting with immune thrombocytopenic purpura: a prospective cohort study. Blood 98: 1760-1764, 2001.

9. Pierrot-Deseilligny Despujol C, Michel M, Khellaf M, et al. Antiphospholipid antibodies in adults with immune thrombocytopenic purpura. $\mathrm{Br} \mathrm{J}$ Haematol 142: 638643, 2008.

10. Sarpatwari A, Bennett D, Logie JW, et al. Thromboembolic events among adult patients with primary immune thrombocytopenia in the United Kingdom General Practice Research Database. Haematologica 95: 1167-1175, 2010.

\section{Correspondence}

Seby John

Cleveland Clinic, 9500 Euclid Avenue

S100-C, Cleveland- 44195, OH, USA

Tel: 216-444-2945

Fax: 216-444-0230

e-mail: johns@ccf.org 